\title{
FASCITIS NECROSANTE
}

Oscar Eduardo Mendoza Ramírez MD.*, Ronald Antonio Medina Lombo MD.**

\section{Resumen}

A pesar de ser una patología con tan baja incidencia, la fascitis necrosante (FN) es importante por sus severas secuelas y elevada tasa de mortalidad. Esta revisión busca ampliar los conocimientos básicos de una enfermedad cuya fisiopatología apenas comienza a ser dilucidada. Existe la falsa creencia de que su devastador comportamiento es secundario a la agresividad de los gérmenes implicados en su etiología, sin embargo su evolución implacable se debe en esencia a la disminución en la perfusión de los tejidos comprometidos, como efecto de la cascada inflamatoria localizada en las fascias que son un espacio desde el punto de vista anatómico susceptible a los cambios de presión tisular. Nuestra revisión busca aclarar conceptos erróneos mediante la exposición detallada de la epidemiología, fisiopatología, clínica, diagnóstico y tratamiento, brindando elementos de valor para que todo el personal médico tanto de pregrado, como profesionales de la salud generales y especialistas, adquiera la capacidad de identificarla y manejarla tempranamente, logrando con ello un importante impacto en su mortalidad.

Palabras clave: fascitis necrosante, infección, necrosis, desbridamiento, fasciotomía.

Abreviaturas: FN, Fascitis necrosante.

\section{Introducción}

La La FN se define como una infección localizada en el tejido celular subcutáneo y la fascia superficial, siendo la representante más agresiva del grupo de las infecciones necrosantes de los tejidos blandos. La fisiopatología de su potente acción destructora ha sido producto de múltiples teorías y aún en nuestra era existen múltiples apreciaciones erróneas que vale la pena desmitificar.

Desde tiempos inmemorables se ha considerado falsamente que la FN es el producto de la infección por gérmenes muy agresivos y multirresistentes. Sin embargo, su difícil manejo tiene que ver más con mecanismos inflamatorios que sumados a la disminución en la perfusión, favorecen el crecimiento bacteriano y disminuyen la respuesta tisular del huésped. Es por tal motivo que el factor que más influye en su desenlace es el diagnóstico y manejo multidisciplinario tempranos, con un tratamiento en el que el antibiótico no es el pilar principal, pues hay que brindar un pronto desbridamiento, adecuado soporte nutricional y cuidado intensivo si es necesario.

Fecha recibido: noviembre 4 de 2006 -Fecha aceptado: diciembre 14 de 2006

* Residente III de patología, Fundación Universitaria de Ciencias de la Salud.

** Residente I de medicina interna, Fundación Universitaria de Ciencias de la Salud.

\section{Historia}

El primer reporte de la enfermedad se debe a Joseph Jones en 1871, un cirujano de la armada de los Estados Unidos que la describió en los soldados que participaron en la guerra civil americana y la llamó gangrena del hospital, ${ }^{1}$ pero existe evidencia que desde el siglo $\mathrm{V}$ antes de nuestra era Hipócrates realizó la primera descripción de lo que hoy conocemos como infecciones necrosantes de los tejidos blandos. ${ }^{2}$ Sin embargo, para el caso en particular de la FN, es en Inglaterra donde fue descrita en forma detallada y caracterizada por el cirujano Leonard Gillespie y los médicos Gilbert Blane y Thomas Trotter de la armada inglesa a finales del siglo XVIII. ${ }^{3}$

En 1924 se identificó por primera vez un germen causal por parte de Meleney, quien debido a sus hallazgos microbiológicos en veinte pacientes en Beijing prefirió llamarla gangrena por Streptococus hemolyticus, ${ }^{4,5}$ y fue desde 1952 que Bob Wilson introdujo el término de FN debido a su signo más ominoso, que es la necrosis de los tejidos blandos localizada en especial a nivel de la fascia. ${ }^{6}$ Durante las últimas décadas esta patología ha recibido innumerables apelativos como infección por "bacterias come carne" y "gangrena galopante", entre otros. ${ }^{3,7}$ 


\section{Epidemiología}

Es imposible estimar en forma precisa los datos estadísticos de la enfermedad debido a la falta de consenso en su nombre y al poco reporte hasta finales del siglo pasado, Su incidencia es relativamente baja, estimada en un 0,4 por 100.000 habitantes, sin embargo existen informes muy variables según la población. ${ }^{8}$ Se han identificado múltiples factores de riesgo dentro de los cuales la diabetes mellitus es la más asociada, además de otras enfermedades crónicas como cirrosis, anemia, cáncer, HIV e insuficiencia renal, ${ }^{9,10}$ estados de inmunocompromiso, alcoholismo, uso de drogas intravenosas, obesidad, enfermedad vascular periférica y tabaquismo. ${ }^{11,12,13}$

La FN puede ocurrir a cualquier edad sin predilección por sexo ${ }^{14}$ se localiza en cualquier parte del cuerpo, ocurriendo con mayor frecuencia en la pared abdominal, las extremidades y el periné, sitio donde se conoce como gangrena de Fournier, ${ }^{15,16,17} 1 \mathrm{la}$ cual fue descrita por Jean Alfred Fournier en $1843 ; ;^{18}$ existen además reportes de casos con localizaciones tan inesperadas como son los párpados. ${ }^{19}$

Debido a que la mayor parte de veces es el resultado de una lesión cutánea que va desde edemas y traumas tan leves como una picadura de insecto, ${ }^{20}$ hasta inyecciones y procedimientos quirúrgicos. ${ }^{11,21,22}$ La enfermedad se ha asociado con infecciones y ulceraciones de la piel, hernias encarceladas, ${ }^{20,21,23}$ obesidad, ${ }^{24}$ cálculos renales ${ }^{16} \mathrm{y}$ lesiones cutáneas producidas por infecciones virales como la varicela que causan una variante llamada varicela gangrenosa. También de ha descrito en pacientes con la piel íntegra como producto de la siembra hematógena de las bacterias o de sus toxinas, o bien en forma de siembra indeterminada que puede ocurrir hasta en un $20 \%$ de los pacientes. ${ }^{21,25,26}$ Para el caso particular de la FN del periné existen múltiples causas dentro de las que se enumeran infecciones urinarias, cálculos uretrales, masaje prostático, instrumentaciones genitourinarias y aún lesiones coitales. ${ }^{17,27,28}$

Se tienen también evidencias muy debatidas respecto a la asociación existente entre el uso de antiinflama- torios no esteroideos (AINES) y el desarrollo de FN en personas jóvenes, previamente sanas que los han recibido como tratamiento para lesiones menores o dolores principalmente articulares. Sin embargo, no existe claridad entre el uso extensivo de estos medicamentos con un desenlace tan poco común como la FN, siendo la tendencia a considerarlo como un evento paralelo y no el producto de una relación causa efecto, a pesar de la clara evidencia de la inducción de inmunosupresión secundaria al uso de dichos fármacos. ${ }^{29,30,31} \backslash$

La mortalidad varía según las diferentes series publicadas y va desde el 6 al $76 \%,{ }^{21}$ siendo la FN del periné la mayor. ${ }^{32}$ Además, se identifican como factores que aumentan la tasa de mortalidad la edad mayor de 60 años, la presencia de dos o más comorbilidades ${ }^{9}$ y el retraso en el diagnóstico y el manejo. ${ }^{13,33,34,35,36}$ Llama la atención que la diabetes a pesar de ser uno de los factores de riesgo más reconocidos no tiene asociación con la mortalidad, al igual que el grado de signos clínicos como el dolor, eritema, edema y el calor en el área comprometida del paciente. ${ }^{9}$

Las principales causas de muerte de los pacientes con FN se atribuyen a falla multiorgánica secundaria a la sepsis en los estadios avanzados de la enfermedad. ${ }^{37}$ Además, hay que tener en cuenta que la mayoría de los pacientes que se recuperan presentan abundantes secuelas tanto físicas como psicológicas, sobre todo a las alteraciones estéticas y a la misma reparación tisular. Dentro de ellas se enumeran el dolor crónico, la ansiedad, la depresión, y las deformidades debidas a amplios desbridamientos y amputaciones. ${ }^{38,39}$

\section{Fisiopatología}

Las infecciones de los tejidos blandos se clasifican según la profundidad de la capa en la que se originan, de manera que pueden comprometer la piel (epidermis y dermis), la dermis y la fascia superficial, las fascias, y los músculos(Gráfica 1). Sin embargo, pueden envolver la totalidad de las capas en estadios avanzados. ${ }^{40} \mathrm{La} \mathrm{FN}$ como su nombre lo indica es la infección que compromete inicialmente las fascias. 
Todos las partes del cuerpo presentan recubrimiento por fascias y en la mayoría de las áreas corporales se reconocen dos, superficial y profunda, las cuales a su vez forman un espacio virtual que separa la piel del tejido muscular; ${ }^{41}$ para el caso particular de las fascias de las extremidades, éstas tienen continuidad con las áreas superiores adyacentes del cuerpo tanto en el tórax como en el periné.

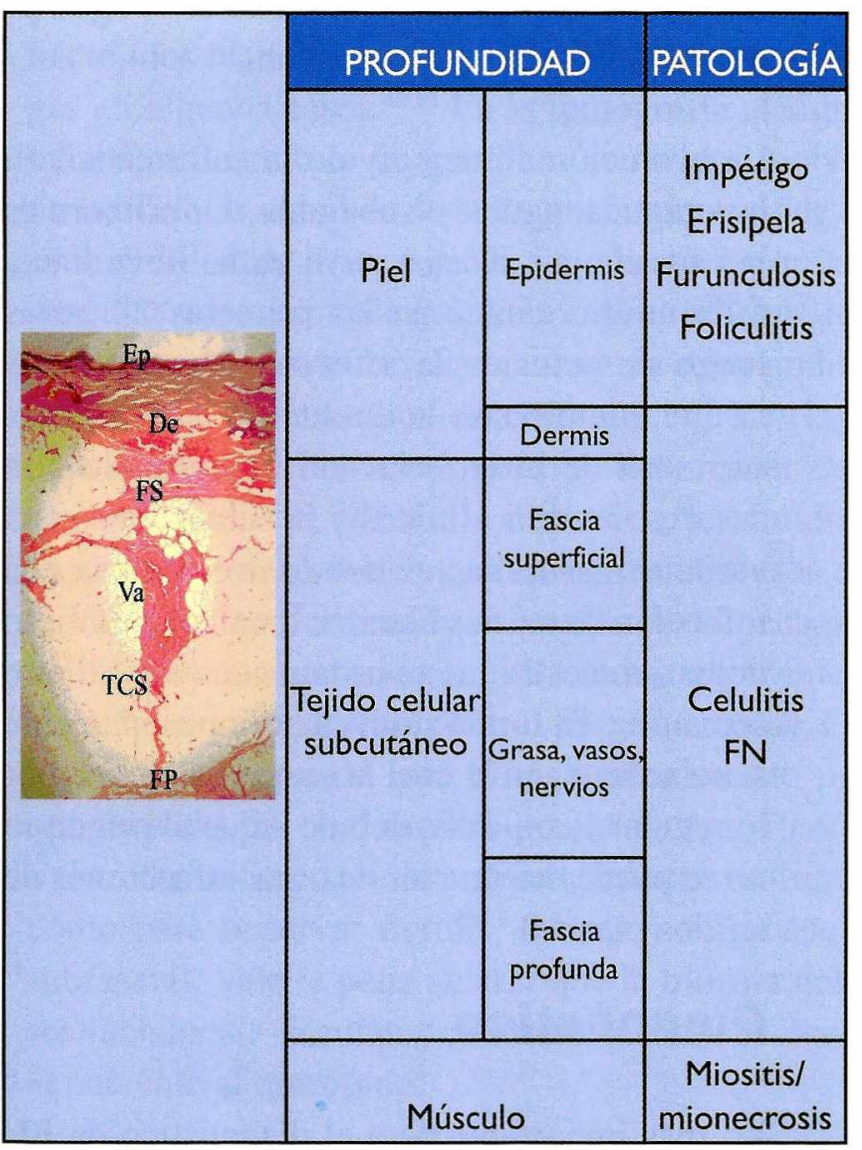

Gráfica I. Clasificación de las infecciones de tejidos blandos. (Ep: epidermis, De: dermis, FS: fascia superficial, Va: vaso, TCS: tejido celular subcutáneo, FP: fascia profunda).

Al microscopio las fascias están compuestas por haces de tejido conjuntivo denso y entre ellas discurren los nervios y abundantes vasos superficiales, los cuales envían ramificaciones perpendiculares a las capas de la superficie. Como ya se enunció, en la mayor parte de los casos se necesita una solución de continuidad de las capas superficiales para permitir el ingreso de gérmenes al espacio ubicado entre las dos fascias, pero las bacterias pueden ingresar por vía hematógena; luego del ingreso de los microorganismos a dicho espacio se disparan los mecanismos de inflamación consistentes en aumento de la perfusión y de la permeabilidad vascular, así como migración de leucocitos. ${ }^{42}$

El disparo de la cascada de la inflamación a nivel del espacio entre las fascias produce un incremento de la presión tisular, lo cual va seguido de una disminución en la perfusión asociada a trombosis secundaria multifactorial, que se ve reflejada en la isquemia progresiva de los tejidos y en el fracaso de los mecanismos de defensa del huésped ante la agresión bacteriana, lo cual que favorece el crecimiento de los microorganismos y sirve de corredor para que el proceso aumente en forma dramática en sentido horizontal a través del espacio infectado (Gráfica 2).

Al contrario de lo que se supone, la FN es el producto de la respuesta inflamatoria localizada en un lugar desde el punto de vista anatómico susceptible al incremento de la presión tisular, sumada al daño producido por una infección de complejidad variable no siempre secundaria a un germen agresivo. ${ }^{11,43,44,45}$ Por tales motivos siempre se debe tener en cuenta que el principal pilar del tratamiento es la disminución de presión en las fascias, lo cual se logra con el desbridamiento quirúrgico o con fasciotomías. ${ }^{13,36}$

\section{Clínica}

Varía según el tiempo de evolución y el grado de inmunocompetencia de los pacientes, pudiéndose enmascarar o modificarse algunos de los síntomas iniciales por comorbilidades de los mismos. La evolución del cuadro se divide en tres estadios clínicos. El primero simula cualquier infección banal d e tejidos blandos y consta de eritema, calor, dolor y edema del área afectada. Cabe resaltar que el dolor siempre es desproporcionado al comparar su intensidad con el compromiso cutáneo aparente.

En el estadio II aparecen vesículas como resultado de la isquemia de los tejidos, signo que es patognomónico de una infección necrosante de tejidos blandos y debe alarmar por la inminencia de una fascitis, ${ }^{46}$ además la piel se indura y puede fluctuar. Por último, 

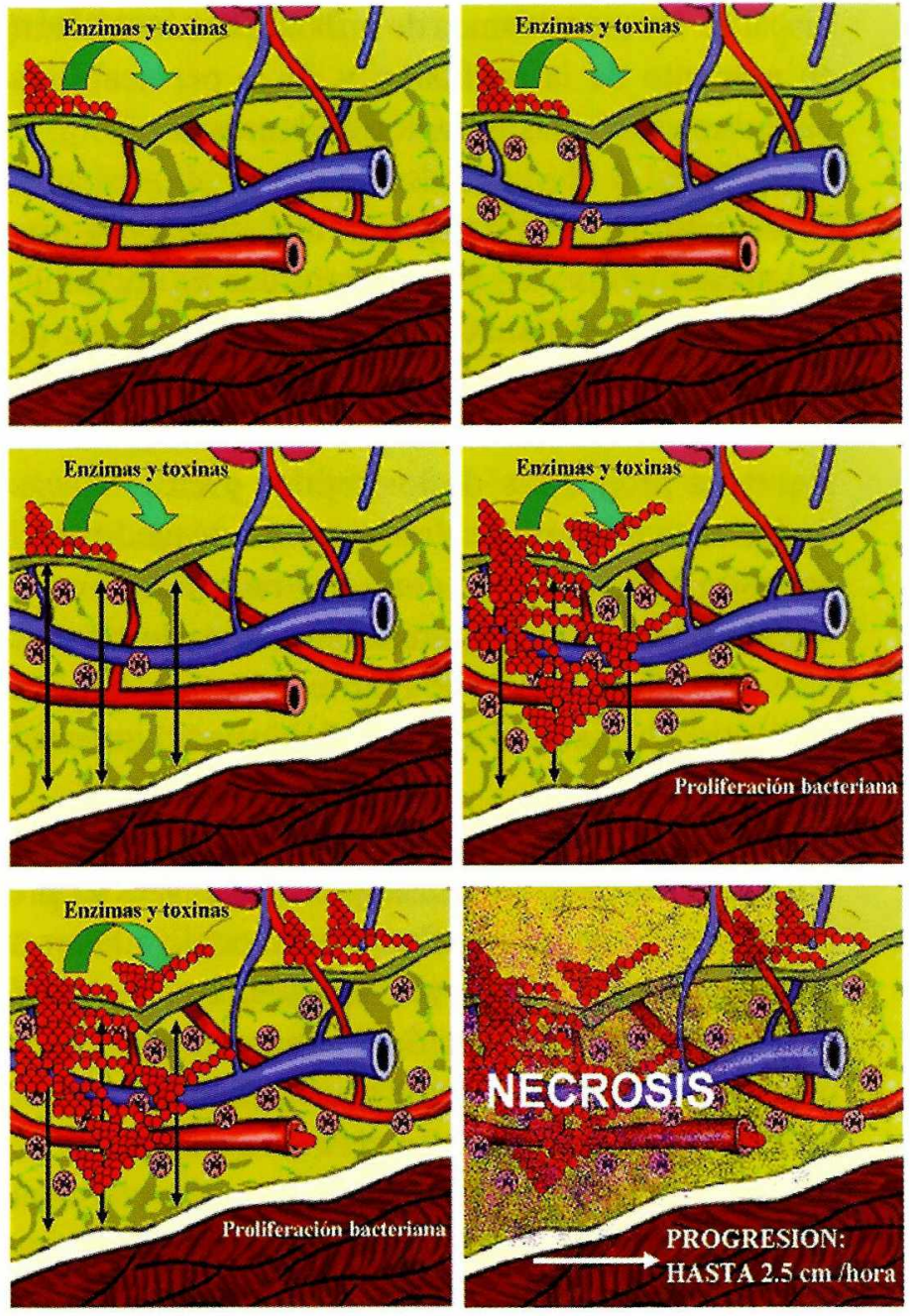

Gráfica 2. Fisiopatología de la FN. La colonización bacteriana del espacio ubicado entre las dos fascias desencadena la cascada inflamatoria produciendo edema, isquemia, trombosis vascular y por último necrosis que progresa en sentido horizontal en forma dramática.

en el estadio III la necrosis se hace evidente, las vesículas se llenan de material hemático, la piel cambia a una coloración violácea, se presenta crepitación y debido a la muerte de los tejidos el dolor desaparece convirtiendo en anestésica al área comprometida. ${ }^{11,33,43,46}$

Además de los síntomas descritos, muchos pacientes debutan con fiebre, hipotensión, falla multiorgánica, alteraciones mentales e incluso choque. ${ }^{8,9,10,21,43}$

\section{Clasificación}

La FN se clasifica de acuerdo con la evolución y los microorganismos implicados en su etiología. Según los gérmenes se divide en tres grupos, el primero es la in- fección polimicrobiana donde actúan de modo sinérgico gram negativos aeróbicos y anaeróbicos, así como gram positivos. El segundo es debido a microorganismos específicos cuyo principal exponente es el Streptococcus beta hemolítico del grupo A, y el tercero es la fascitis causada por Vibrios marinos que se adquieren por la punción con un pez o marisco o por la exposición de una herida o picadura al agua de mar. $9,47,48,49,50$

La evolución clínica divide la enfermedad en hiperaguda, aguda y subaguda. La primera es la causada por Vibrios cuyo curso lleva a una falla multiorgánica en las primeras 24 horas luego de ocurrida la infección. La aguda es la que cumple con la evolución clínica antes expuesta de la enfermedad cumpliendo con sus tres estadios clínicos y la subaguda es una variante más indolente donde se evidencia una infección de tejidos blandos focal, con mínimo dolor o molestia que se instaura en varios días o semanas y en forma súbita deteriora al paciente si no se trata, en la cual la sospecha clínica por lo regular es mínima, debido a que al principio no se puede diferenciar de otras infecciones de tejidos blandos. ${ }^{43,45}$

\section{Diagnóstilco}

Lo más importante para el diagnóstico de $\mathrm{FN}$ es la sospecha clínica, sumada a otros hallazgos como los resultados de paraclínicos e imagenología, los datos de patología y algunas otras herramientas como la prueba del dedo y la saturación de oxígeno del tejido comprometido.

Existen estudios que avalan la realización de pruebas de laboratorio para aumentar la sospecha diagnóstica. El más importante se conoce como LRINEC (The Laboratory Risk Indicator for Necrotizing Fasciitis Store) (Tabla 1), en el cual se analizaron trece variables y se determinó que existen seis independientes que según su resultado dan un diagnóstico con alta sensibilidad pero baja especificidad, observando un 
valor predictivo positivo del $40 \%$ y uno negativo del $95 \%$, lo cual obliga a practicar otras pruebas que confirmen el diagnóstico, pues aunque los resultados de el LRINEC sean consistentes con la presencia de FN cabe la posibilidad de encontrarse ante otra severa infección de tejidos blandos. . $^{10,43,50,51,52,53}$

En cuanto a imágenes diagnósticas, los rayos $\mathrm{X}$ pueden revelar aumento de la opacidad y espesor de los tejidos blandos con la posibilidad de evidenciar gas en algunos casos. ${ }^{49,54}$ En la tomografía el signo más significativo es la aparición de fluidos a nivel de las fascias, además de su engrosamiento y de la probabilidad de franca necrosis con compromiso vascular o presencia de gas. Vale la pena resaltar que en algunos pacientes la falla renal aguda contraindica la utilización de medios de contraste. ${ }^{49,55}$ La resonancia magnética nuclear permite determinar con mayor claridad el edema, la necrosis y los fluidos, facilitando diferenciar la celulitis y los abscesos de la FN, pero no delimita la extensión de la necrosis por el edema encontrado en los tejidos adyacentes. 49,56,57. La prueba de oro para el diagnóstico de FN es la realización de una biopsia idealmente por congelación, la cual medirá por lo menos $10 \times 7$ x 7 milímetros y será lo suficientemente profunda como para observar dermis, fascias y el músculo subfascial. Vale la pena aclarar que la biopsia debe ser incisional, mediante anestesia local, sin llevar al paciente al quirófano.

Se realizará en estadios iniciales en los que exista duda diagnóstica, pues cuando la necrosis se encuentra instaurada no se pueden diferenciar los hallazgos histopatológicos de los observados en otras infecciones severas de tejidos blandos y además retrasa el manejo quirúrgico con un importante impacto en la mortalidad. ${ }^{34,58}$

A nivel microscópico se evidencia edema, inflamación severa de la dermis y el tejido celular subcutáneo con necrosis y hemorragia focales asociadas, hiperplasia intimal, vasculitis, trombosis vascular y colonias bacterianas ${ }^{49,58}$ (Graficas 3, 4 y 5). Contrario a lo que se supone, no existe aval para la determinación microbiológica del germen en los
TABLA I. LRINEC (THE LABORATORY RISK INDICATOR FOR NECROTIZING FASCITIS SCORE)

\begin{tabular}{|c|c|c|}
\hline Creatinina & $<1.5 \mathrm{mg} / \mathrm{dl}$ & 0 \\
\hline & $>1.5 \mathrm{mg} / \mathrm{dl}$ & 2 \\
\hline \multirow[t]{3}{*}{ Recuento leucocitario } & $<15.000 / \mathrm{mm} 3$ & 0 \\
\hline & $15.000-25.000 / \mathrm{mm} 3$ & 1 \\
\hline & $>25.000 / \mathrm{mm} 3$ & 2 \\
\hline \multirow[t]{3}{*}{ Hemoglobina } & $>13.5 \mathrm{~g} / \mathrm{dl}$ & 0 \\
\hline & $11.13 .5 \mathrm{~g} / \mathrm{dl}$ & 1 \\
\hline & $<\| \mathrm{l} g / \mathrm{dl}$ & 2 \\
\hline \multirow[t]{2}{*}{ Sodio } & 135 o más mEq/L & 0 \\
\hline & $<135 \mathrm{mEq} / \mathrm{L}$ & 2 \\
\hline \multirow[t]{2}{*}{ PCR } & $<150 \mathrm{mg} / \mathrm{dl}$ & 0 \\
\hline & $>150 \mathrm{mg} / \mathrm{dl}$ & 4 \\
\hline \multirow[t]{3}{*}{ Glicemia } & 180 o menos mg/dl & 0 \\
\hline & $>180 \mathrm{mg} / \mathrm{dl}$ & 1 \\
\hline & RIESGO BAJO & $\begin{array}{c}5 \text { ó } \\
\text { MENOS }\end{array}$ \\
\hline RESULTADO & $\begin{array}{l}\text { RIESGO } \\
\text { INTERMEDIO }\end{array}$ & 607 \\
\hline
\end{tabular}

tejidos de la biopsia estudiada por patología, por lo cual se recomienda realizar cultivos en una muestra independiente en laboratorio clínico. ${ }^{47}$

Para los casos donde no exista la posibilidad de biopsia por congelación se puede efectuar la prueba del dedo, que consiste en una incisión que permita llegar a las fascias y luego de su identificación se intenta la disección de forma roma, considerando la prueba como positiva cuando se pierde la resistencia 


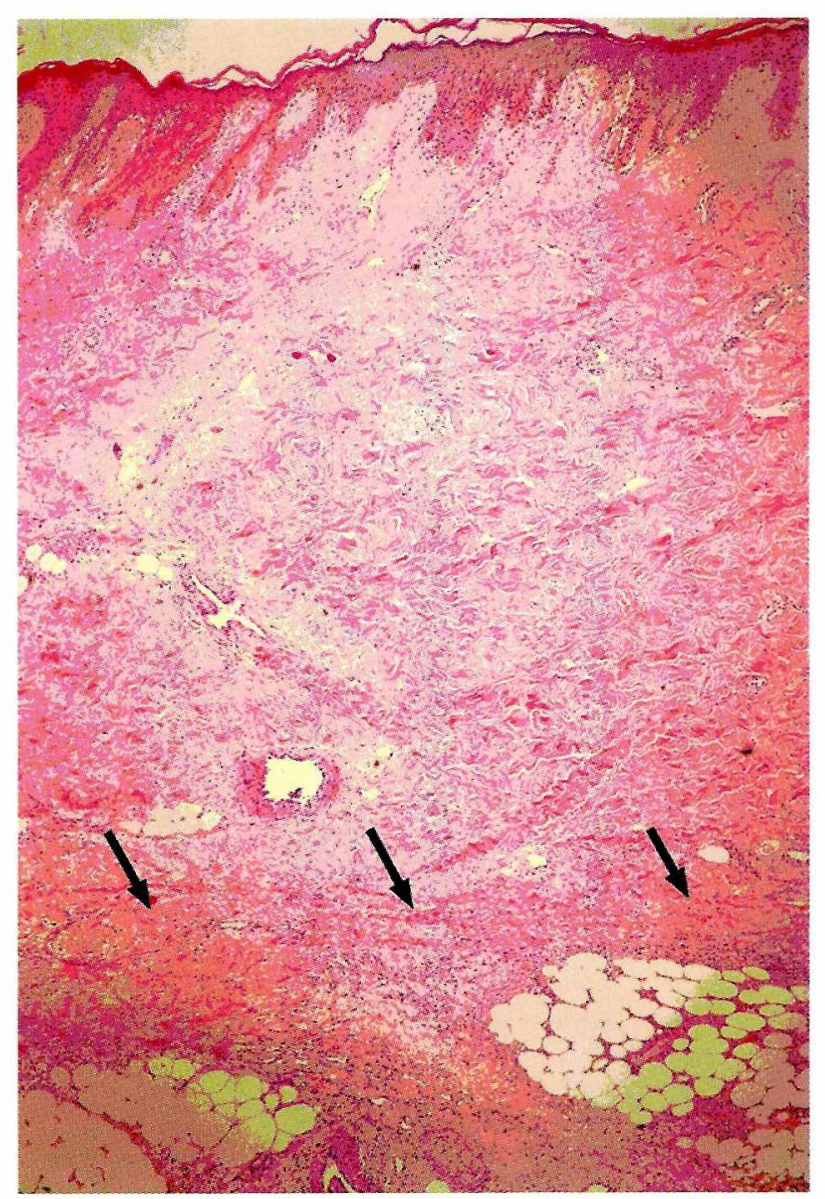

Gráfica 3. Imagen histológica de un cuadro de fascitis en estadios iniciales. Obsérvese la fascia superficial (flechas) y el infiltrado inflamatorio mixto a nivel de los tejidos profundos.

del tejido, el sangrado es ausente o hay presencia de material seropurulento adyacente a la fascia; sin embargo, es una prueba que requiere experiencia y no debe ser intentada por novatos dada su alta subjetividad. ${ }^{11,43,50,51}$

Por último, existen otras posibilidades para confirmar la FN cuya eficacia aún no es clara. Se puede medir la saturación de oxígeno a nivel de los tejidos comprometidos y compararla con los tejidos sanos, encontrando diferencias del $30 \%$ en promedio con recuperación de la saturación posterior a la fasciotomía, lo cual confirma su valor diagnóstico. Sin embargo se debe tener en cuenta que pueden existir factores que interfieran en los resultados como estasis venosa crónica, lesiones vasculares periféricas, choque, hipoxia, etc. ${ }^{59}$

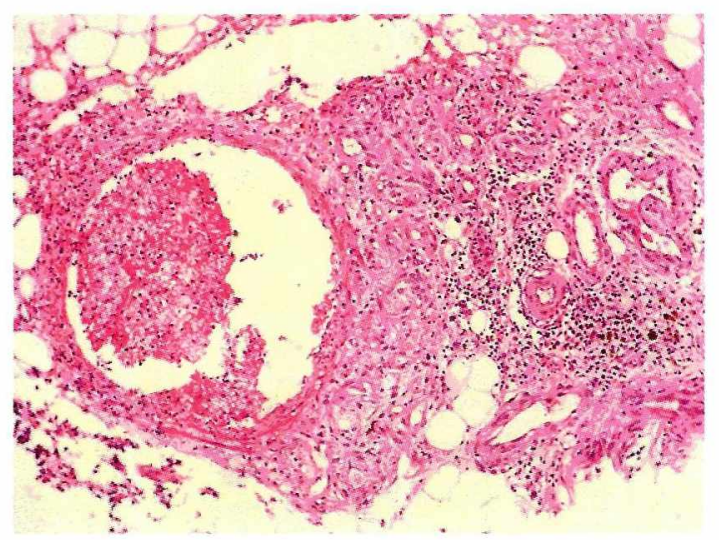

Gráfica 4. Imagen histológica que evidencia inflamación mixta con presencia de un trombo reciente intravascular y necrosis focal del tejido adyacente.

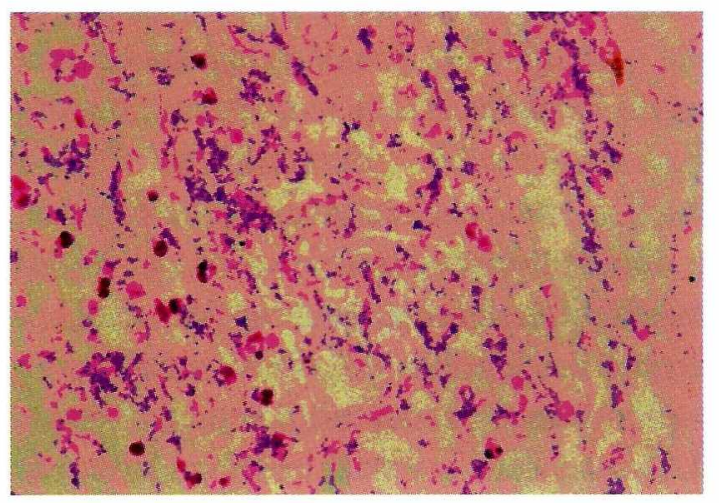

Gráfica 5. FN en estadio avanzado en que se observan abundantes colonias bacterianas con polimorfonucleares asociados y necrosis severa. No existe diferencia histopatológica con otras infecciones necrosantes de tejidos blandos severas o en fases avanzadas.

Dentro de las posibilidades emergentes se encuentran la medición de los niveles de citoquinas y los estudios moleculares para detectar los gérmenes en los tejidos, pero son métodos diagnósticos con muy bajo acceso y con escasa evidencia a favor. ${ }^{60,61}$

\section{Tratamiento}

El tratamiento de la FN comienza por la sospecha y diagnóstico temprano de la patología por parte de médico tratante inicial, ya que su oportuno diagnóstico y la instauración de un manejo precoz guardan proporciones directas con la mortalidad. ${ }^{13,33,34,35,36}$

Dicho manejo debe enfocarse de acuerdo con la naturaleza de la enfermedad considerándola siempre como una verdadera catástrofe infecciosa secundaria a una isquemia progresiva de los tejidos y a una falla 
en los mecanismos de defensa del paciente, por lo tanto debe tenerse siempre presente que el tiempo es el factor fundamental en la búsqueda de la supervivencia, encontrando diferencias significativas en la mortalidad cuando su diagnóstico y tratamiento se instauran en las primeras 24 horas, al compararlas con aquellos casos en los que se inicia luego del primer día de iniciados los síntomas. ${ }^{58}$

Las premisas fundamentales del tratamiento de la FN son cirugía precoz, antibioticoterapia, soporte de cuidado intensivo y, si es posible, terapia con oxigeno hiperbárico. ${ }^{62,63}$ Una vez el diagnóstico de fascitis se ha establecido, el tratamiento del paciente debe realizarlo un equipo multidisciplinario que incluye los servicios de cirugía general y cirugía plástica, medicina critica e infectología (Tabla 2) ${ }^{63,64,65}$

El personal del área quirúrgica debe encargarse del desbridamiento temprano o de la realización de fasciotomías, ya que es la principal arma en el tratamiento de la FN, pues incluso con el adecuado manejo antibiótico se requiere contar con la perfusión de los tejidos para permitir la penetración de los agentes terapéuticos en el área afectada. ${ }^{45} \mathrm{El}$ desbridamiento quirúrgico se considera ideal cuando realiza excisión completa del tejido necrótico con establecimiento de drenaje quirúrgico amplio de colecciones y con la subsecuente hemostasia de los bordes que deben sangrar como signo indirecto de su adecuada perfusión. ${ }^{62}$

Los pacientes deben ser llevados a lavados y ampliaciones de desbridamientos si se evidencia progresión de la necrosis en el examen diario del tejido. Incluso se debe sopesar la posibilidad de amputar el miembro afectado si no se logra su control, en especial en pacientes con enfermedad vascular periférica o diabetes. $^{48}$

El equipo de infectología debe realizar un análisis de cada caso e iniciar un adecuado régimen de antibióticos en búsqueda del cubrimiento de la diversidad y variedad de los microorganismos implicados, siempre con tinción de Gram y con cultivos en medios aeróbico y anaeróbico en progreso, y realizar ajuste del mismo según los reportes de laboratorio obtenidos de los cultivos. ${ }^{62,63,64}$

El manejo antimicrobiano empírico en los casos en que se sospecha que la infección se debe a Streptococcus grupo A se recomienda el uso de clindamicina (600-900 mg cada 6 a 8 horas) asociado con penicilina $\mathrm{G}$ ( 4 millones de unidades cada 4 horas)

\section{Tabla I. Pasos a seguir ante la sospecha de Fascitis necrotizante}

I.Historia clínica completa y examen físico.

2. Laboratorios (LRINEC) y/o estudios imagenológicos como herramientas diagnósticas.

3.Interconsulta con infectología.

4.Interconsulta con cirugía.

5.Biopsia por congelación en estadios iniciales sospechosos.

6.Cultivos y tinción de Gram para aerobios anaerobios.

7.Soporte y vigilancia hemodinámica por parte de cuidado crítico.
8. Reanimación con fluidos, estabilización cardiovascular y preparación preoperatoria.

9. Antibióticos endovenosos antes de acto quirúrgico.

10.Profilaxis antitetánica.

II.Completo desbridamiento de tejido necrótico en acto quirúrgico inicial.

12.Soporte nutricional en postoperatorio.

13. Vigilancia hemodinámica en postoperatorio.

14.Valoración periódica (segun el caso) y modificación nmediata el manejo si el estado del paciente lo requiere. 
como primera combinación terapéutica. Como segunda opción se puede utilizar clindamicina a la misma dosis asociada con cefalosporinas de primera o segunda generación.

En la FN con sospecha de gérmenes mixtos (aerobios y anaerobios) se recomienda el uso de ampicilina ( 2 gr iv cada 4 horas), asociada con clindamicina (600-900 mg iv cada 6 a 8 horas) y a ciprofloxacina (400 mg iv cada 6 a 8 horas) como primera opción terapéutica; vancomicina ( 1 gr iv cada 6 horas), asociada con metronidazol ( $500 \mathrm{mg}$ iv cada 6 horas) y a ciprofloxacina ( $400 \mathrm{mg}$ iv cada 6 a 8 horas) como segunda opción de tratamiento. ${ }^{66} \mathrm{Se}$ resalta que el uso de ciprofloxacina es discutido en ambiente hospitalario por su alta tasa de generación de resistencia, lo cual la deja a criterio del equipo tratante.

El servicio de cuidado crítico se encarga de realizar reanimación con cristaloides e inotropos y/o vasopresores en búsqueda de alcanzar las metas de reanimación establecidas en el protocolo de Rivers para el manejo de choque séptico y la sepsis. ${ }^{62,65}$ Además, deben proveer un adecuado soporte nutricional y velar por la detección temprana de infecciones nosocomiales agregadas y controlar las fallas presentadas en los sistemas orgánicos. ${ }^{35,48,62}$

El manejo de cada enfermo por parte del equipo multidisciplinario debe ser dinámico, modificando de inmediato la antibioticoterapia, el soporte de cuidado intensivo y nutricional o realizando intervenciones quirúrgicas más allá de lo presupuestado en el abordaje inicial si es necesario, dejando a un lado las consideraciones estéticas siempre bajo la premisa de que se maneja un cuadro que atenta contra la vida del paciente. ${ }^{64}$

Otra de las posibilidades terapeúticas con eficacia demostrada es el oxígeno hiperbárico, pues la terapia a tres atmósferas es bactericida incluso para el Clostridium perfringens y bacteriostático para otra gran variedad de bacterias. Además, la tensión de oxígeno inhibe la producción de alfa toxina del clostridium y mejora la acción bactericida de los leucocitos polimorfonucleares. Existe evidencia de que eleva la sensibilidad a los antibióticos como sucede con la tobramicina en las infecciones por Pseudomona aeuriginosa, el trimetroprim sulfametoxazol y la nitrofurantoina cuyo efecto es prolongado por la hiperoxia in vitro. Se ha demostrado que el uso de oxígeno hiperbárico en infecciones no clostridianas disminuye la mortalidad de $66 \%$ a $23 \%$ y de 1.2 a 3.3 el requerimiento de desbridamientos posteriores. ${ }^{67,68,69}$

\section{Conclusiones}

La FN es una enfermedad necrosante de tejidos blandos cuyo desencadenante es la presencia de bacterias o toxinas bacterianas en las fascias, lo cual lleva al disparo de la cascada inflamatoria con aumento secundario de la presión tisular e hipoperfusión asociada, permitiendo el sobrecrecimiento bacteriano y el avance implacable de la infección a través de dicho espacio.

Es por tal motivo que se recomienda tener especial atención a los cuadros atípicos de infecciones de tejidos blandos, con una alta sospecha clínica seguida del uso adecuado de las herramientas diagnósticas que permitan su confirmación, para así poder brindar un tratamiento temprano y afectar de forma positiva su alta tasa de mortalidad así como sus secuelas físicas y psicológicas.

\section{Referencias}

1. Jones J. Investigation on the nature, causes and treatment of hospital gangrene as it prevails in the confederate armies 1861-1865. En: Hasting Hamilton F, editor. Surgical memoirs of the war rebellion. New York: Sanitary commission; 1871 .

2. Descamps V, Aitken J, Lee MG. Hippocrates on necrotising fasciitis. Lancet. 1994; 344 (8921): 556.

3. Loudon I. Necrotising fasciitis, hospital gangrene, and phagedena. Lancet. 1994; 344: 1416-19.

4. Meleney FL. Hemolytic streptococcus gangrene. Arch Surg. 1924; 9: 317-364.

5. Meleney FL. A differential diagnosis between certain typers of infectious gangrene of the skin. Surg Gynecol Obstet. 1933; 56: 847-67. 
6. Wilson B. Necrotizing fasciitis. Am Surg. 1952; 18(4) : 416-31.

7. Nowak R. Flesh-eating bacteria: not new, but still wirrisome. Science. 1994; 264: 1665.

8. Trem JT, Kirshner RS. Diagnosis necrotizing fasciitis. Adv Skin Wound Care. 2002; 15: 135-38.

9. Yuag-Meng L, Chih-Yu C, et al. Microbiology and factors affecting mortality in necrotizing fasciitis. J Microbiol Immunol Infect. 2005; 38: 430- 35.

10. Wall DB, De Virgilio C, et al. Objective criteria may assist in distinguishing necrotizing fasciitis soft tissue infection. Am J Surg. 2000; 179 (1): 17-21.

11. Schroeder JL, Steinke EE. Necrotizing fasciitis - the imporance of early diagnosis and debridement. AORN J. 2005; 82(6): 1031-40.

12. Canoso J, Barza M. Soft tissue infections. Rheum Dis Clin North Am. 1993; 19: 293- 309.

13. Voros D, Pissiotis C, et al. Role of early and extensive surgery in the treatment of severe necrotizing soft tissue infections. Br J Surg. 1993; 80: 1190-91.

14. Chelsom J, Halstensen A, et al. Necrotising fasciitis due to group A streptococci in western Norway: incidence and clinical features. Lancet. 344 (8930):1111-5.

15. Umbert IJ, Winkelmann RK, et al. Necrotizing fasciitis: a clinical, microbiologic, and histopathologic study of 14 patients. J Am Acad Dermatol. 1989; 20(5 Pt 1):774-81.

16. Ward RG, Walsh MS. Necrotizing fascitis: 10 years' experience in district general hospital. J Am Acad Dermatol. 1991; 78: 488-89.

17. Stephens BJ, Lathrop JC, et al. Fournier's gangrene: historic (1764-1978) versus contemporany (1979-1988) differences in etiology and clinical importance. Am Surg. $1993 ; 59: 149-54$.

18. Fournier JA. Gangrene foudroyante de la verge. Sem Med. 1883; 3: 345-47.

19. Kronish JW, McLeish WM. Eyelid necrosis and periorbital necrotizing fasciitis. Report of a case and review of the literature. Ophthalmology. 1991; 98(1):92-8.

20. Sudarsky LA, Laschinger JC, et al. Improved results from a standardized approach in treating patients with necrotizing fascitis. Ann Surg. 1987; 206: 661-65.

21. McHenry CR, Piotrowski JJ, et al. Determinants of mortality in necrotizing soft tissue infections. Ann Surg. 1995; 221: 558-63.

22. Francis KR, Lamaute HR, et al. Implication of risk factors in necrotizing fasciitis. Am Surg. 1993; 559: 304-08.

23. Andze G, Pagbe JJ, et al. Postoperative necrotizing fasciitis in infants. Apropos of a case. J Chir (Paris). 1995 Feb; 132(2):90-3.

24. Angelici AM, Nasti AG, et al. Necrotizing fasciitis: our experience. G Chir. 2004; 25(5):167-70.

25. Schwarz G, Sagy M, Barzilay Z. Multifocal necrotizing fasciitis in varicella. Pediatr Emerg Care. 1989; 5: 31-3.

26. McHenry CR, Brandt CP, et al. Idiopathic necrotizing fascitis: recognition, incidence, and outcome of therapy. Am Surg. 1994; 60: 490-94.

27. Laucks SS. Fournier's gangrene. Surg Clin North Am.1994; 74: $1339-52$.

28. Efem SE. The features and etiology of Fournier's gangrene. Postgrad Med. 1994; 70: 568-71.

29. Rimailho A, Riou B, et al. Fulminant necrotizing fasciitis and nonsteroidal anti-inflammatory drugs. J Infect Dis. 1987; 55: 143-46.

30. Brun-Buisson CJL, Saada M, et al. Haemolytic streptococcal gangrene and nonsteroidal anti-inflammatory drugs. BMJ. 1985; 290: 1786.

31. Van Ammers P, Moore P, Sacho H. Necrotizing fasciitisafter caesarean section - association with nonsteroidal antiinflammatory drugs. S Afr Med J. 1991; 80: 203-04.

32. Maynor M. "Necrotizing fascitis". E-medicine [serie en Internet]. [citado 15 Ene. 2005]: [aprox 3 p.]. Disponible en: http://www.emedicine.com/emerg/topic332.htm.

33. Wong $\mathrm{CH}$, Chang $\mathrm{HC}$ et al. Necrotizing fasciitis: clinical presentation, microbiology, and determinants of mortality. J Bone Joint Surg Am. 2003; 85-A (8): 1454-60.

34. Majeski JA, Majeski E. Necrotizing fasciitis: improved survival with early recognition by tissue biopsy and aggressive surgical treatment. South Med J. 1997; 90: 1065-68.

35. Majeski JA, Alexander JW. Early diagnosis, nutricional support and immediate extensive debridement improve survival in necrotizing fasciitis. Am J Surg. 1983; 145: 781-87.

36. Bilton BD, Zibari GB, et al. Aggressive surgical management of necrotizing fasciitis serves to decrease mortality a retrospective study. Am Surg. 1998; 64: 397-401.

37. Walshaw C, Deans H. CT findings in necrotizing fasciitis: a report of four cases. Clin Radiol. 1996; 51: 429-32.

38. Faucher LD, Morris SE, et al. Burn center management of necrotizing soft-tissue surgical infections in unburned patients. Am J Surg. 2001; 182(6): 563-69.

39. Elliott DC, Kufera, JA, et al. Necrotizing soft tissue infections: risk factors for mortality and strategies for management. Ann Surg. 1996; 224(5): 672- 83.

40. Conly J. Soft tissue infections. En: Hall JB, Schmidt GA, et al. Principles of critical care. New Cork: Mc Graw-Hill; 1992. p. 1325-34. 
41. Moore, K. Anatomía con orientación clínica. 3a ed. Madrid: Médica Panamericana; 1993.

42. Ramsi C, Vinay K, et al. Patología estructural y funcional. 6a ed. Filadelfia: McGraw-Hill; 2000.

43. Wong C, Wang Y. The diagnosis of necrotizing fasciitis. Curr Opin Infect Dis. 2005; 18: 101-06.

44. Wilkerson R, Paul W, et al. Necrotizing fasciitis: review of literature ans case report. Clin Orthop Relat. 1987; 216: 187-92.

45. Barker F, Leppard B, Seal D. Streptococcal necrotizing fasciitis: comparison between histological and clinical features. J Clin Pathol. 1987; 40: 335-41.

46. Seal D. Necrotizing fasciitis. Curr Opin Infect Dis. 2001; 14: $127-32$.

47. Guiliano A, Lewis F, et al. Bacteriology of necrotizing fasciitis. Am J Surg. 1977; 134: 52-7.

48. Green R, Dafoe D, et al. Necrotizing fasciitis. Chest. 1996; 110: 219-27.

49. Fuggit J, Puckett M, et al. Necrotizing fasciitis. Radiographics. 2004; 24(5): 1472-76.

50. Barie $\mathrm{P}$. The laboratory risk indicator for necrotizing fasciitis (LRINEC) score: useful tool or paralysis by analisis? Crit Care Med. 2004; 32(7): 1618-19.

51. Wong C, Khin L, et al. The LRINEC (The Laboratory Risk Indicator for Necrotizing Fasciitis) score: a tool for distinguishing necrotizing fasciitis from other soft tissue infections. Crit Care Med. 2004; 32(7): 1535-41.

52. Wong C. Clinical relevance of the LRINEC (The Laboratory Risk Indicator for Necrotizing Fasciitis) score for assessment of early Necrotizing Fasciitis. Crit Care Med. 2005; 33(7):1677.

53. Wall, D, Klein, S, et al. A simple model to help distinguish necrotizing fasciitis from nonnecrotizing soft tissue infection. J Am Coll Surg. 2000; 191(3): 227-31.

54. Struk D, Punk P, et al. Imaging of soft tissue infections. Radiol Clin North Am. 2001; 39: 277-301.

55. Becker M, Zbaren $\mathrm{P}$, et al. Necrotizing fasciitis of the head and neck: role of CT in diagnosis and management. Radiology. 1997; 202: 471-76.

56. Millar T, Randolph D, et al. Fat-suppressed MRI of mus- culoskeletal infection: fast T2-weighted images techniques versus gadolinium-enhanced T1-weighted images. Skeletal Radiol. 1997; 26: 654-58.

57. Brothers T, Tagle D, et al. Magnetic resonance imaging differentiates between necrotizing fasciitis and non-necrotizing fasciitis of the lower extremity. J Am Coll Surg. 1998; 187(4): 416-21.

58. Stamenkovic I, Lew D. Early recognition of potentially fatal necrotizing fasciitis. N Engl J Med. 1984; 310(26): 1689-93.

59. Wang T, Hung C. Role of tissue oxygen saturation monitoring in diagnosing necrotizing fasciitis of the lower limbs. Ann Emerg Med. 2004; 44(3): 222-28.

60. Rodríguez R, Abdullah R, et al. A pilot study of citokine levels and white blood cell counts in the diagnosis of necrotizing fasciitis. Am J Emerg Med. 2006; 24: 58-61.

61. Muldrew K, Simpson J, et al. Molecular diagnosis of necrotizing fasciitis by $16 \mathrm{~S}$ rRNA gene sequencing and superantigen gene detection. J Mol Diagn. 2005; 7(5): $641-45$.

62. Majeski J, John J. Necrotizing soft tissue infections: a guide to early diagnosis and initial therapy. South Med J. 2003; 96(9):900-05.

63. Praba-Egge A, Lanning D, et al. Necrotizing fasciitis of the chest and abdominal wall arising from an empyema, J Trauma. 2004; 56:1356-61.

64 Patiño JF. Infecciones necrotizantes de la piel y de los tejidos blandos: guías para el manejo de urgencias. Bogotá: Ministerio de Protección Social; 2002.

65. Rivers E, Nguyen B, Havstad S, et al. Early goal-directed therapy in the treatment of severe sepsis and septic shock. N Engl J Med. 2001; 345(19): 1368-77.

66. Wu W, Lieber M. Hyperbaric oxygen therapy: ten common questions related to the management of severe necrotizing skin and soft-tissue infections. Infect Dis Clin Pract. 2001; $10: 429-34$

67.Wilkinson D; Doolette, et al. Hyperbaric medicine unit, department of anaesthesia and intensive care. Arch Surg. 2004; 139(12):1339-45.

68. Riseman J, Zamboni W, et al. Hyperbaric oxygen therapy for necrotizing fasciitis reduces mortality and the need for debridements. Surgery. 1990; 108(5): 847-50.

69. Harrison T, Fauci A. Principios de medicina interna. 16a ed. [Madrid]: Interamericana; 2006.

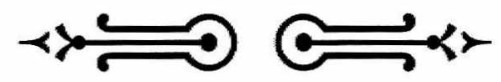

